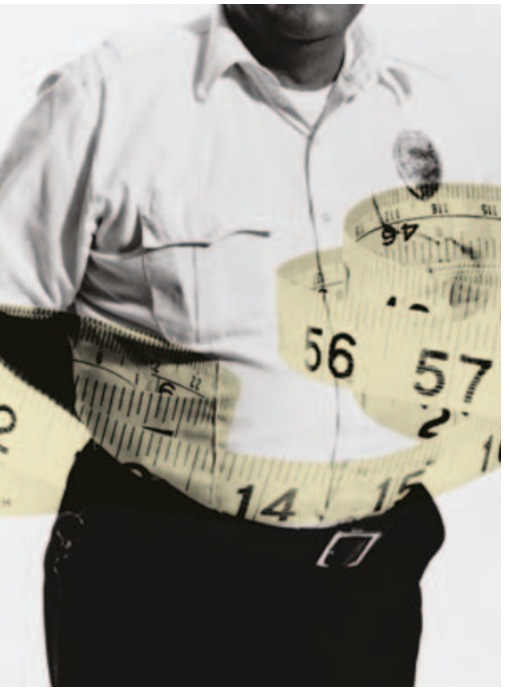

Proof of the pudding: should patients be diagnosed as having metabolic syndrome?

\title{
Groups question existence of metabolic syndrome
}

Study says people should not be diagnosed with the condition.

\section{Simon Frantz}

Two of the biggest diabetes organizations have stirred up a hornet's nest by arguing that there is not enough scientific evidence to justify diagnosing patients with metabolic syndrome.

Researchers from the American Diabetes Association (ADA) and the European Association for the Study of Diabetes (EASD) say that metabolic syndrome is poorly defined, inconsistently used and that there is considerable doubt regarding its value as a cardiovascular risk marker (Kahn, R. et al. Diabetes Care 28, 2289-2304; 2005).

'Metabolic syndrome' was originally coined as a term to provide a helpful reminder that there is a clustering of cardiovascular disease risk factors whose underlying pathophysiology is thought to be related to insulin resistance, says Richard Kahn, Chief Scientific and Medical Officer for the ADA, and co-author of the paper. "But the term metabolic syndrome has taken on a meaning that is greater than is justified by our current knowledge," says Kahn.

There is no evidence that individual risk factors, such as blood pressure, triglyceride or cholesterol levels, act synergistically, and therefore each risk factor should be treated on its own aggressively, conclude the authors. "Our knowledge base is such that we cannot yet contemplate drug treatment for insulin resistance, let alone the metabolic syndrome," they write.

The problem with confining this to individual risk factors is that from the cardiovascular perspective we look at the whole patient, says Scott Grundy, Director of the Center for Human Nutrition at the University of Texas Southwestern Medical Center at Dallas. "We went through the single-risk-factor treatment idea in the cardiovascular field; we argued that issue for many years, and we're fairly evolved to the point that the total risk factor of the patient needs to be taken into consideration for drug treatment."

To say that the major mechanisms are unknown is not strictly true, as obesity is a major driving force behind the prevalence of this clustering, and diet and exercise is known to reduce risk, argues Grundy, who co-authored the just-published American Heart Association (AHA) and the National Heart, Lung, and Blood Institute (NHLBI) guidelines on diagnosing and treating metabolic syndrome (Grundy S. M., et al. Circulation published online 12 Sept 2005; doi:10.1161/CIRCULATIONAHA.105.169405)

. "Finding out how these risk factors play out in the body, I agree that's for future research - but does that mean we shouldn't deal with an obese patient that has those risk factors?"
Under the new AHA/NHLBI guidelines, patients should be considered as having metabolic syndrome if they have abnormal levels of three of the following factors: abdominal obesity; triglyceride levels; highdensity lipoprotein cholesterol; blood pressure; and fasting blood glucose.

However, these aren't the only guidelines. Recent guidelines by the International Diabetes Federation, meant to replace existing World Health Organization guidelines, are very similar to the AHA/NHLBI formulation but do differ in diagnostic criteria requirements.

"The message [from the ADA and EASD paper] that further research needs to be done to really nail down which of these different criteria are the more important ones is a valid point," says David Moller, who leads the Metabolic Disorders discovery unit at Merck Research Laboratories. "But from my perspective, I think that having some definition of metabolic syndrome is better than having none."

Additional prospective studies with drugs that treat one or more of the risk factors are needed to show which factors are important. Retrospective research could also be carried out, with drug companies mining through their databases of trials of patients with lipid disorders or diabetes, says Moller. All of which would be easier if there was a consensus on how to define the metabolic syndrome.

Prospective studies are already under way to examine the effects of diet and exercise in patients with impaired glucose tolerance, many of whom fulfill the criteria for the metabolic syndrome. But prospective studies examining whether drug therapies can prevent cardiovascular disease in people with the metabolic syndrome are also needed, says Neil Ruderman, Chief of Diabetes and Metabolism at Boston University Medical Center.

The concept of the metabolic syndrome has succeeded in raising awareness of obesity and inactivity as the causes of many diseases - and diet and exercise in their prevention - says Ruderman. "I believe the vast majority of the medical community believes in the entity of the metabolic syndrome, even if we still cannot define it precisely and our understanding of the biochemical mechanism(s) that underlies it is incomplete," he says.

\footnotetext{
"The term metabolic syndrome has taken on a meaning that is greater than is justified by our current knowledge."
} 